\title{
THE NONEXISTENCE OF SOME NONCOMPACT CONSTANT MEAN CURVATURE SURFACES
}

\author{
LEUNG-FU CHEUNG
}

(Communicated by Peter $\mathrm{Li}$ )

\begin{abstract}
Using isoperimetric inequality, we prove that there are no complete noncompact surfaces in $\mathbb{R}^{3}$ with finite total curvature, odd Euler characteristic, and mean curvature bounded away from zero.
\end{abstract}

\section{INTRODUCTION}

This note is originated from the observation that the existence of an isoperimetric inequality on a complete noncompact surface implies the unboundedness of the surface area. This motivated Theorem 2.1, which says that complete noncompact connected surfaces in $\mathbb{R}^{3}$ of finite curvature and odd Euler characteristic must have quadratic area growth. As a consequence, we show that there are no complete noncompact constant mean curvature surfaces with finite total curvature and odd Euler characteristic in $\mathbb{R}^{3}$, unless they are minimal. In the case of minimal surfaces, there are a lot of nontrivial examples of complete surfaces satisfying the assumptions of the theorem, such as the Euclidean plane (total curvature $=0$ ), the Enneper's surface (total curvature $=-4 \pi$ ), the second order Enneper-like surfaces given by the Weierstrass data $f=1$; $g=z^{2}$ (total curvature $=-8 \pi$ ), etc. This is our first step in understanding whether there are noncompact constant mean curvature surfaces with fintie total curvature or finite area at all.

We would like to thank Professor Peter Li for his very kind and helpful suggestions.

\section{Results}

Throughout the following, a surface is said to have finite total curvature if the $L_{2}$ norm of its second fundamental form $B$ is finite.

Theorem 2.1. Let $M$ be an oriented complete noncompact surface with finite total curvature immersed in $\mathbb{R}^{3}$. Then it either has quadratic growth or even Euler characteristic (or equivalently an even number of ends).

The proof of the theorem is based on the following theorem of White and a formula (Lemma 2.3) related to the isoperimetric inequality.

Received by the editors October 7, 1992 and, in revised form, November 2, 1992.

1991 Mathematics Subject Classification. Primary 53A10, 53C42. 
Lemma 2.2 (White [W]). Let $M$ be a complete noncompact surface immersed in $\mathbb{R}^{3}$ such that the total curvature is finite. Then

$$
\int_{M} K d M=4 m \pi, \quad \text { where } m \text { is an integer. }
$$

Lemma 2.3 (Li and Tam [LT]). Let $M$ be a complete noncompact surface satisfying $\int_{M} K_{-}<\infty$, where $K_{-}$denotes the negative part of the Gaussian curvature. Then the following holds:

$$
2 \lim _{r \rightarrow \infty} \frac{A(r)}{r^{2}}=2 \pi \chi-\int_{M} K d M,
$$

where $A(r)$ denotes the area of the geodesic ball of radius $r$ centered at a given point $p$.

Proof of Theorem 2.1. Since the surface has finite total curvature, the assumptions for Lemma 2.2 and Lemma 2.3 are satisfied. Hence, on one hand we have $\int_{M} K d M=4 \pi m$, for some integer $m$, and on the other hand, $\int_{M} K d M=$ $2 \pi \chi-2 \lim _{r \rightarrow \infty} r^{-2} A(r)$. Combining these two equations, we obtain

$$
\chi-2 m=\lim _{r \rightarrow \infty} \frac{A(r)}{\pi r^{2}},
$$

hence if $\chi$ is odd then the area of the surface has quadratic growth.

Remark. Theorem 2.1 implies that a surface with finite total curvature which is embedded near infinity in $\mathbb{R}^{3}$ has quadratic area growth at each end. This can be seen as follows. Since the surface is embedded near infinity, there is an embedded simple closed curve on each end beyond which the surface is a half-cylinder. Cut the surface along each of these curves and complete the ends obtained by adding immersed disks along the curves. Now one can apply Theorem 2.1 to conclude that each of the ends has quadratic area growth.

Corollary 2.4. Let $M$ be a complete noncompact surface immersed in $\mathbb{R}^{3}$ with finite total curvature and odd Euler characteristic. Then $\nexists \varepsilon \geq 0$ such that $|H| \geq$ $\varepsilon>0$.

Remark. The set of complete noncompact surfaces in $\mathbb{R}^{3}$ with odd Euler characteristic, finite total curvature, and $|H| \geq \varepsilon>0$ is nonempty, even in the case of simply connected constant mean curvature surface. In this case, one can find examples in the associated family to the Delaunay surface (see Do Carmo and Dajzer [CD]).

Remark. Corollary 2.4 implies in particular that there are no simply connected complete noncompact constant mean curvature surfaces of finite total curvature if it is not a minimal surface.

Proof of Corollary 2.4. Since the surface has odd Euler characteristic, Theorem 2.1 implies that its area is unbounded, i.e., $A(M)=\infty$. On the other hand, the hypothesis $\exists \varepsilon>0,|H| \geq \varepsilon>0$ implies $\infty>\int_{M}|B|^{2} d M \geq 2 \int_{M} H^{2} d M \geq$ $2 \int_{M} \varepsilon d M=2 A(M)=\infty$, hence one gets the required contradiction. 


\section{REFERENCES}

[CD] M. Do Carmo and M. Dajzer, Helicoidal surfaces with constant mean curvature, Tôhoku Math. J. 34 (1982), 425-435.

[LT] P. Li and L. F. Tam, Complete surfaces with fintie total curvature, J. Differential Geom. 33 (1991), 139-168.

[W] B. White, Complete surfaces of finite total curvature, J. Differential Geom. 26 (1987), 315-326.

Department of Mathematics, National University of Singapore, 10 Kent Ridge CresCENT, SINGAPORE 0511, SINGAPORE 